\title{
THERMODYN AMIC PROPERTIES OF SOLUBILITY OF 2-METHYL-5-ARYLFURAN-3-CARBOXYLIC ACIDS IN ORGANIC SOLVENTS
}

\author{
Iryna Sobechko ${ }^{1, *}$, Volodymyr Dibrivnyi $^{1}$, Yuri Horak $^{2}$, Nadiia Velychkivska $^{3}$, \\ Victoriia Kochubei $^{1}$, M ykola Obushak ${ }^{2}$
}

https://doi.org/10.23939/chcht11.04.397

\begin{abstract}
The temperature dependencies of both acids solubility in acetonitrile, dimethylketone, isopropanol, ethylacetate and benzene were investigated. The results were represented by Shredder's equation according to which enthalpies, entropies and Gibbs energy of solubility at $298 \mathrm{~K}$ were determined. Fusion heats of the acids were determined using differential thermal analysis. In accordance with obtained values the enthalpies, entropies and Gibbs energy of mixing were calculated. The compensating effect of mixing was observed for al solvents with carboxy group.
\end{abstract}

Keywords: enthalpy, entropy, Gibbs energy of solubility, mixing and fusion, 2-methyl-5-phenylfuran-3-carboxylic acid, 2-methyl-5-(4-methylphenyl)-furan-3-carboxylic acid.

\section{Introduction}

Arylfuran derivatives are important compounds from a practical standpoint because such structural fragments are part of many natural and synthetic substances that exhibit biological activity [1-2]. Some of them are used as drugs, in particular for the treatment of neurodegenerative diseases such as stroke, Parkinson's disease, and Alzheimer's disease [3]. Therefore, the synthesis and study of properties of new materials containing arylfuryl fragment is a topical problem.

One of the best approaches to the synthesis of functionalized arylfurans is Meerwein reaction [4-8].

Recently functionalized arylfurans were used in multicomponent reactions allowing to save resources and

\footnotetext{
${ }^{1}$ Lviv Polytechnic National University, S.Bandery St., 12, 79013 Lviv, Ukraine;

${ }^{2}$ Ivan Franko National University of Lviv, Kyryla i Mefodiya St. 6, 79005 Lviv, Ukraine

${ }^{3}$ Institute of Macromolecular Chemistry AS CR, v.v.i., 2 Heyrovskeho Sq., 16206 Prague, Czech Republic

* phys.chem.lp@gmail.com

(c) Sobechko I., Dibrivnyi V., Horak Y., Velychkivska N., Kochubei V., Obushak M., 2017
}

promote the concept of "green chemistry" [9]. Until now the studies were conducted by trial method. Determination of thermodynamic parameters of compounds with arylfuran fragment will allow to define energetic properties of their molecules and energy costs for the synthesis, purification and application. At the same time, the mentioned processes are carried out in solvent medium. So the study of thermodynamic solubility of the compounds with the arylfuran fragment is necessary for the processes optimization.

This work is a continuation of our previous works to determine the thermodynamic solubility of acids, aldehydes and their derivatives in a number of solvents [10-13]. The aim of the presented work is to investigate thermodynamic solubility of 2-methyl-5-phenylfuran-3carboxylic and 2-methyl-5-(4-methylphenyl)-furan-3carboxylic acids in the organic solvents.

\section{Experimental}

\subsection{Investigated Compounds}

Synthesis of 2-methyl-5-phenylfuran-3-carboxylic and 2-methyl-5-(4-methylphenyl)-furan-3-carboxylic acid was carried out according to the scheme (Fig. 1) in three stages.

At the first stage ethyl-2-acetyl-4-oxo-4-arylbutanones (3) were obtained via mixing ethylacetoacetate (1), metallic sodium suspension in toluene and phenacylbromide (2). At the second stage the obtained compounds reacted with phosphorus pentoxide in the medium of benzene and formed ethyl esters of 5-(4methyl)aryl-2-methyl-3-furancarboxylic acids (4). At the third stage 2-methyl-5-(4-methyl)-phenylfuran-3carboxylic acid (5) was obtained via saponification of the synthesized esters (4). The resulting colorless crystals of acids (5) were filtered and recrystallized several times from ethanol. The samples taken after $4^{\text {th }}$ and $5^{\text {th }}$ recrystallization were used. 


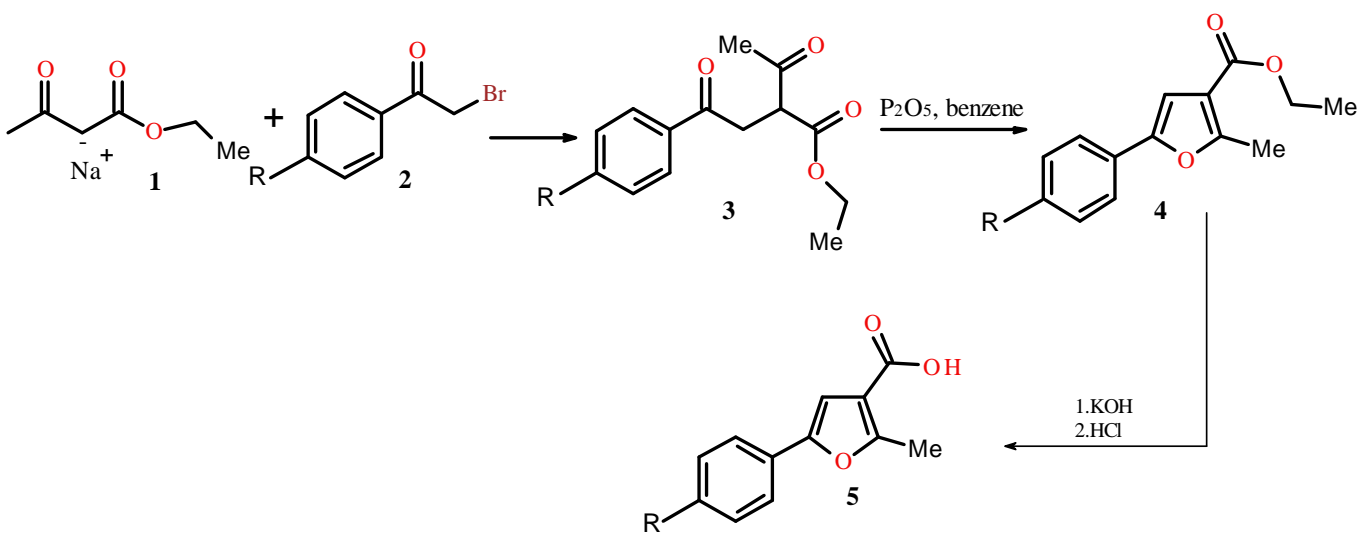

Fig. 1. Scheme of 2-methyl-5-(4-methyl)phenylfuran-3-carboxylic acids synthesis

Table 1

Physico-chemical properties of the solvents

\begin{tabular}{|l|c|c|c|c|c|c|}
\hline \multirow{2}{*}{\multicolumn{1}{|c|}{ Solvents }} & \multirow{2}{*}{$M, \mathrm{~g} / \mathrm{mol}$} & \multicolumn{2}{|c|}{$n_{D}^{20}$} & \multicolumn{2}{c|}{$T_{\text {boil }}, \mathrm{K}$} & \multirow{2}{*}{$\begin{array}{c}\text { Content of the main } \\
\text { component, wt.\% }\end{array}$} \\
\cline { 3 - 6 } & & lit. & determ. & lit. & determ. & 99.9 \\
\hline Acetonitrile & 41.053 & $1.3442[15]$ & 1.3444 & $354.8[15]$ & 354.6 & 99.8 \\
\hline Benzene & 78.114 & $1.5017[15]$ & 1.5016 & $353.2[15]$ & 353.0 & 99.9 \\
\hline Ethylacetate & 88.106 & $1.3724[15]$ & 1.3722 & $350.2[15]$ & 349.9 & 99.8 \\
\hline Acetone & 58.080 & $1.3591[15]$ & 1.3590 & $329.3[15]$ & 329.2 & 99.9 \\
\hline Isopropanol & 60.096 & $1.3776[15]$ & 1.3776 & $355.3[15]$ & 355.1 & \\
\hline
\end{tabular}

The samples were identified by means of elemental analysis for carbon and hydrogen content, as well as NMR spectroscopy. ${ }^{1} \mathrm{H}$ NMR spectra for 2-methyl-5-phenylfuran-3-carboxylic acid were recorded by Varian 600 instrument (600 MHz), and for 2-methyl-5-(4-methylphenyl)-furan-3-carboxylic acid - by Bruker DRX $500(500 \mathrm{MHz})$. DMSO-d6 was a solvent. Chemical shifts $(\delta, \mathrm{ppm})$ are given relative to DMSO signal (2.50 ppm).

2-Methyl-5-phenylfuran-3-carboxylic acid (Fig. 2): ${ }^{1} \mathrm{H}$ NMR (600 MHz, DMSO) $\delta 2.65\left(\mathrm{~s}, 3 \mathrm{H}, \mathrm{CH}_{3}\right), 7.16(\mathrm{~d}$, $\mathrm{J}=3.3 \mathrm{~Hz}, 1 \mathrm{H}$, fur), $7.31\left(\mathrm{t}, \mathrm{J}=8.1 \mathrm{~Hz}, 1 \mathrm{H}, \mathrm{C}_{6} \mathrm{H}_{5}\right), 7.55$ $\left(\mathrm{d}, \mathrm{J}=8.0 \mathrm{~Hz}, 2 \mathrm{H}, \mathrm{C}_{6} \mathrm{H}_{5}\right), 7.81\left(\mathrm{~d}, \mathrm{~J}=8.0 \mathrm{~Hz}, 2 \mathrm{H}, \mathrm{C}_{6} \mathrm{H}_{5}\right)$, 11.09 (bs, $\mathrm{COOH}$ ).

2-Methyl-5-(4-methylphenyl)-furan-3-carboxylic acid (Fig. 2): ${ }^{1} \mathrm{H}$ NMR (500 MHz, DMSO) $\delta 2.23$ (s, 3H, $\mathrm{CH}_{3} \mathrm{C}_{6} \mathrm{H}_{4}$ ), 2.61 (s, 3H, $\mathrm{CH}_{3}$ fur), 6.88 ( $\mathrm{s}, 1 \mathrm{H}, 4-\mathrm{H}$ fur), $7.26\left(\mathrm{~d}, 2 \mathrm{H}, \mathrm{J}=8,4 \mathrm{~Hz}, 2,6-\mathrm{H} \mathrm{C}_{6} \mathrm{H}_{4}\right), 7.52(\mathrm{~d}, 2 \mathrm{H}$, $\left.\mathrm{J}=8,6 \mathrm{~Hz}, 3,5-\mathrm{H} \mathrm{C}_{6} \mathrm{H}_{4}\right), 12.39$ (s, $\left.1 \mathrm{H}, \mathrm{COOH}\right)$.

Purity of the acids samples obtained in the fourth and fifth stages of their recrystallization was indirectly confirmed by coincidence (within the experimental error) of the fusion enthalpies temperatures calculated by DTA.

The substances significantly different by polarity were used as the solvents: acetonitrile, benzene, acetone, isopropanol, and ethyl acetate produced by Merks company. The content of the main component was at least
$99.0 \mathrm{wt} \%$. Before using, the solvents were additionally purified by fractional distillation followed by identification relative to refractive index and boiling point. The content of the main component was determined by LHM-8D gas-liquid chromatograph with thermalconductivity detector. Chromatograph columns with the length of $2 \mathrm{~m}$ and diameter of $0.4 \mathrm{~cm}$ were filled by Chromator N-AW solid phase with the particles of $0.20-0.25 \mathrm{~mm}$. The solid phase contained $10 \%$ of polyethylene glycol adipate $+1 \%$ of phosphoric acid. Gas carrier was helium. The temperatures of columns and evaporator were 423 and $493 \mathrm{~K}$, respectively. The current of thermal-conductivity detector was $120 \mathrm{~mA}$.

The difference between the resulting values of physico-chemical properties of investigated solvents and literature data was within the experimental error, and the content of the main component was no less than 99.8 wt \% (Table 1 ).

\subsection{Dissolution}

Here it is impossible to determine the enthalpies of dissolution using calorimetry as it was done in [14-16], because investigated compounds have very low dissolution. That is why we used gravimetry, the same as in $[17,18]$. 
Acids were dissolved in a hermetic glass container equipped by Teflon stirrer and thermometer. The dissolution temperature was maintained using water thermostat within $\pm 0.1 \mathrm{~K}$. The rotation speed of the stirrer was $50 \mathrm{rev} / \mathrm{min}$. The solutions were saturated for $48 \mathrm{~h}$ without stirring and $1 \mathrm{~h}$ under constant stirring. To prove the establishment of equilibrium the investigations were carried out at increasing and decreasing temperatures. In our previous experiments we found that under the mentioned conditions the significant change in acids solubility disappeared after 40-45 min for all solvents. Therefore, the solution saturation was performed for 60 min under constant (50 rev/min) stirring. To confirm the attainment of equilibrium the experiments were conducted both by temperatures increasing and lowering. The absence of hysteresis loop on the curve of solubility depending on temperature confirmed near the equilibrium state.

The solutions $(0.6-0.8 \mathrm{~g})$ were selected by a series of two or three samples. Then they were transferred to cups and the solvent was removed at 333-343 K. The cups were weighted to determine the weight of solution and dry residue with the accuracy of $\pm 0.0002 \mathrm{~g}$.

\subsection{Differential Thermal Analysis}

Enthalpy of acids fusion was determined by differential thermal analysis data using Q-1500 D derivatograph of Paulik-Paulik-Erdey system. The samples were analyzed under dynamic mode with the heating rate of $3 \mathrm{~K} / \mathrm{min}$ in the air.

Derivatograph cells were calibrated using standard samples of diphenyl $(3.016 \pm 0.032 ; \quad 343.1 \pm 0.3 \mathrm{~K})$ benzoic acid $(3.413 \pm 0.046 ; 396.8 \pm 0.5 \mathrm{~K})$, adipic acid
$(3.827 \pm 0.042 ; \quad 424.4 \pm 0.4 \mathrm{~K}), \quad$ and silver nitrate $(4.584 \pm 0.089 ; 484.3 \pm 0.4 \mathrm{~K})$. The values in brackets are the coefficients of heat transfer $(\mathrm{J} / \mathrm{K} \cdot \mathrm{s})$ at fusion temperature of the substances. The temperature dependence of heat transfer coefficient is described by second degree polynomial (10):

$$
\begin{gathered}
K \cdot 10^{2}=3.668-1.128 \cdot 10^{-2} T+2.723 \cdot 10^{-5} T^{2} ; \\
S^{2}=5.96 \cdot 10^{-7}
\end{gathered}
$$

More information about derivatograph calibration is presented in [10].

\section{Results and Discussion}

Primary results of investigated acids solubility are represented in Table 2, where weight of dissolved compound $\left(m_{2}\right)$, solubility in mole fractions $\left(X_{2}\right)$ and temperature $(T)$ under which the solubility was determined are listed. The experimental data were processed using least-squares method and represented in the same Table as a linear form of Shredder's equation (1):

$$
\ln X_{2}=-\Delta_{\text {sol }} H / R T+\Delta_{\text {sol }} S / R
$$

where acid solubility $X_{2}$ relates to the average temperature of the investigated temperature interval $\Delta T ; \Delta_{\text {sol }} H$ and $\Delta_{\text {sol }} S$ - partial molar enthalpy and entropy of solubility. Hereinafter all errors are given for the significance level of 0.95 .

Initially the investigated acids are crystalline substances, so to calculate their partial molar enthalpies and entropies of mixing $\left(\Delta_{\text {mix }} H, \Delta_{\text {mix }} S\right)$ it is necessary to determine the corresponding enthalpy and entropy of fusion $\left(\Delta_{\text {fus }} H, \Delta_{\text {fus }} S\right)$.

Table 2

The temperature dependence of 2-methyl-5-phenylfuran-3-carboxylic acid and 2-methyl-5- (4-methylphenyl)-furan-3-carboxylic acid solubility in organic solvents

\begin{tabular}{|c|c|c|c|c|c|c|c|c|c|}
\hline$T, \mathrm{~K}$ & $m_{2} \cdot 10^{2}, \mathrm{~g}$ & $X_{2} \cdot 10^{2}$ & $T, \mathrm{~K}$ & $m_{2} \cdot 10^{2}, \mathrm{~g}$ & $X_{2} \cdot 10^{2}$ & $T, \mathrm{~K}$ & $m_{2} \cdot 10^{2}, \mathrm{~g}$ & $X_{2} \cdot 10^{2}$ \\
\hline 1 & 2 & 3 & 4 & 5 & 6 & 7 & 9 \\
\hline \multicolumn{7}{|c|}{ 2-methyl-5-phenylfuran-3-carboxylic acid } \\
\hline \multicolumn{7}{|c|}{ Acetonitrile } \\
\hline 297.4 & 0.61 & 0.29 & 304.9 & 0.76 & 0.35 & 314.1 & 1.11 & 0.52 \\
\hline 297.4 & 0.66 & 0.28 & 304.9 & 0.76 & 0.35 & 314.1 & 1.12 & 0.52 \\
\hline 297.4 & 0.72 & 0.29 & 308.5 & 0.79 & 0.40 & 316.5 & 1.11 & 0.54 \\
\hline 299.0 & 0.71 & 0.30 & 308.5 & 0.92 & 0.40 & 316.5 & 1.14 & 0.53 \\
\hline 299.0 & 0.79 & 0.30 & 308.5 & 1.18 & 0.40 & 316.5 & 1.23 & 0.53 \\
\hline 299.5 & 0.64 & 0.29 & 309.4 & 0.82 & 0.40 & 316.9 & 1.09 & 0.53 \\
\hline 299.5 & 0.68 & 0.29 & 309.4 & 0.96 & 0.40 & 316.9 & 1.15 & 0.54 \\
\hline 299.5 & 0.77 & 0.29 & 309.4 & 1.22 & 0.41 & 316.9 & 1.16 & 0.53 \\
\hline 300.4 & 0.68 & 0.33 & 312.1 & 0.94 & 0.45 & 319.2 & 1.21 & 0.60 \\
\hline 300.4 & 0.73 & 0.34 & 312.1 & 0.95 & 0.45 & 319.2 & 1.48 & 0.60 \\
\hline 300.4 & 0.81 & 0.34 & 312.1 & 0.98 & 0.45 & 320.5 & 1.15 & 0.60 \\
\hline 304.4 & 0.68 & 0.34 & 313.5 & 0.90 & 0.49 & 320.5 & 1.51 & 0.61 \\
\hline 304.4 & 0.71 & 0.34 & 313.5 & 0.94 & 0.49 & 323.5 & 1.48 & 0.68 \\
\hline
\end{tabular}


Table 2 (continued)

\begin{tabular}{|c|c|c|c|c|c|c|c|c|}
\hline 1 & 2 & 3 & 4 & 5 & 6 & 7 & 8 & 9 \\
\hline 304.4 & 0.85 & 0.35 & 313.5 & 1.13 & 0.48 & 323.5 & 1.56 & 0.68 \\
\hline 304.9 & 0.72 & 0.35 & 314.1 & 0.94 & 0.51 & 323.5 & 2.53 & 0.67 \\
\hline \multirow{2}{*}{\multicolumn{9}{|c|}{$\frac{\ln X_{2}=(4.93 \pm 0.42)-(3218 \pm 131) \cdot 1 / T ; \rho=0.9825 \%}{\text { Acetone }}$}} \\
\hline & & & & & & & & \\
\hline 278.3 & 2.63 & 1.72 & 292.0 & 3.64 & 2.32 & 309.4 & 3.94 & 3.46 \\
\hline 278.3 & 3.11 & 1.72 & 292.0 & 3.75 & 2.34 & 309.4 & 4.48 & 3.44 \\
\hline 278.3 & 3.74 & 1.71 & 292.0 & 3.87 & 2.34 & 309.4 & 5.95 & 3.47 \\
\hline 279.0 & 2.36 & 1.79 & 298.9 & 3.58 & 2.91 & 313.5 & 5.50 & 3.79 \\
\hline 279.0 & 2.39 & 1.74 & 298.9 & 3.96 & 2.83 & 313.5 & 6.09 & 3.79 \\
\hline 279.0 & 2.54 & 1.78 & 298.9 & 4.92 & 2.83 & 313.5 & 9.56 & 3.76 \\
\hline 279.0 & 2.62 & 1.75 & 303.5 & 4.09 & 3.18 & 318.5 & 6.58 & 4.24 \\
\hline 279.0 & 2.77 & 1.77 & 303.5 & 4.33 & 3.15 & 318.5 & 6.75 & 4.23 \\
\hline 279.0 & 3.08 & 1.75 & 303.5 & 5.57 & 3.15 & 318.5 & 9.33 & 4.21 \\
\hline 287.4 & 3.05 & 2.11 & 304.2 & 3.88 & 3.05 & 323.0 & 7.51 & 4.74 \\
\hline 287.4 & 3.26 & 2.10 & 304.2 & 4.29 & 3.02 & 323.0 & 8.57 & 4.77 \\
\hline 287.4 & 3.30 & 2.11 & 304.2 & 5.26 & 3.03 & 323.0 & 10.32 & 4.78 \\
\hline \multicolumn{9}{|c|}{$\ln X_{2}=(3.11 \pm 0.14)-(1998 \pm 43) \cdot 1 / T ; \rho=0.9962 \%$} \\
\hline & & & & sopropar & & & & \\
\hline 280.7 & 1.01 & 1.01 & 304.7 & 3.94 & 2.05 & 314.0 & 4.83 & 2.59 \\
\hline 280.7 & 1.27 & 0.99 & 304.7 & 4.59 & 2.04 & 318.1 & 3.47 & 2.89 \\
\hline 280.7 & 1.72 & 0.99 & 308.6 & 1.87 & 2.23 & 318.1 & 4.19 & 2.84 \\
\hline 290.2 & 1.71 & 1.31 & 308.6 & 2.62 & 2.15 & 320.0 & 4.24 & 3.16 \\
\hline 290.2 & 1.79 & 1.31 & 308.6 & 3.99 & 2.14 & 320.0 & 4.39 & 3.15 \\
\hline 290.2 & 2.75 & 1.27 & 312.0 & 2.38 & 2.37 & 320.0 & 5.07 & 3.15 \\
\hline 297.0 & 2.02 & 1.49 & 312.0 & 3.28 & 2.38 & 322.6 & 3.95 & 3.55 \\
\hline 297.0 & 2.06 & 1.48 & 312.0 & 3.85 & 2.37 & 322.6 & 5.35 & 3.55 \\
\hline 297.0 & 2.14 & 1.50 & 313.4 & 3.89 & 2.53 & 322.6 & 5.74 & 3.52 \\
\hline 304.0 & 2.61 & $\begin{array}{l}1.87 \\
\end{array}$ & 313.4 & 3.93 & 2.55 & 323.2 & 5.72 & 3.48 \\
\hline 304.0 & 3.13 & 1.90 & 313.4 & 4.98 & 2.54 & 323.2 & 6.32 & 3.45 \\
\hline 304.0 & 3.20 & 1.91 & 314.0 & 2.64 & 2.58 & 323.2 & 7.18 & 3.45 \\
\hline 304.7 & 3.92 & 2.04 & 314.0 & 4.60 & 2.60 & & & \\
\hline \multicolumn{9}{|c|}{$\ln X_{2}=(5.04 \pm 0.33)-(2728 \pm 102) \cdot 1 / T ; \rho=0.9880 \%$} \\
\hline & & & & thylacet & & & & \\
\hline 278.1 & 1.88 & 1.49 & 303.8 & 3.78 & 2.70 & 313.5 & 4.34 & 3.39 \\
\hline 278.1 & 1.96 & 1.50 & 308.0 & 3.59 & 2.93 & 313.5 & 6.19 & 3.41 \\
\hline 278.1 & 2.62 & 1.49 & 308.0 & 4.08 & 2.94 & 315.5 & 3.78 & 3.59 \\
\hline 289.4 & 2.13 & 1.98 & 308.0 & 5.17 & 2.92 & 315.5 & 4.49 & 3.57 \\
\hline 289.4 & 2.38 & 1.99 & 308.0 & 2.65 & 3.04 & 315.5 & 4.76 & 3.56 \\
\hline 289.4 & 2.27 & 1.99 & 308.0 & 3.50 & 3.03 & 319.7 & 4.10 & 3.88 \\
\hline 293.6 & 2.76 & 2.12 & 308.0 & 4.31 & 3.03 & 319.7 & 4.23 & 3.82 \\
\hline 293.6 & 3.17 & 2.17 & 309.1 & 2.62 & 3.06 & 319.7 & 5.40 & 3.86 \\
\hline 293.6 & 3.24 & 2.16 & 309.1 & 3.07 & 3.05 & 320.0 & 4.39 & 3.92 \\
\hline 296.1 & 2.66 & 2.30 & 309.1 & 4.21 & 3.06 & 320.0 & 4.69 & 3.90 \\
\hline 296.1 & 2.71 & 2.31 & 313.0 & 2.43 & 3.36 & 320.0 & 7.05 & 3.86 \\
\hline 296.1 & 3.55 & 2.32 & 313.0 & 3.03 & 3.38 & 323.1 & 4.63 & 4.34 \\
\hline 303.8 & 3.51 & 2.69 & 313.0 & 3.84 & 3.36 & 323.1 & 4.83 & 4.32 \\
\hline 303.8 & 3.58 & 2.69 & 313.5 & 3.85 & 3.42 & 323.1 & 5.68 & 4.31 \\
\hline \multicolumn{9}{|c|}{$\ln X_{2}=(3.23 \pm 0.13)-(2074 \pm 41) \cdot 1 / T ; \rho=0.9962 \%$} \\
\hline \multicolumn{9}{|c|}{ Benzene } \\
\hline 281.5 & 0.75 & 0.37 & 308.2 & 1.04 & 0.94 & 313.5 & 2.11 & 1.26 \\
\hline 281.5 & 0.78 & 0.36 & 308.2 & 1.19 & 1.03 & 317.9 & 1.67 & 1.36 \\
\hline 281.5 & 0.80 & 0.35 & 308.2 & 1.21 & 0.95 & 317.9 & 1.73 & 1.33 \\
\hline 298.0 & 0.78 & 0.64 & 308.6 & 1.29 & 0.89 & 317.9 & 2.13 & 1.44 \\
\hline 298.0 & 0.90 & 0.69 & 308.6 & 1.39 & 0.95 & 322.5 & 1.66 & 1.67 \\
\hline 298.0 & 1.00 & 0.68 & 308.6 & 1.73 & 0.92 & 322.5 & 1.80 & 1.50 \\
\hline 304.0 & 0.98 & 0.85 & 313.1 & 1.54 & 1.11 & 322.5 & 1.85 & 1.51 \\
\hline 304.0 & 1.09 & 0.81 & 313.1 & 1.57 & 1.09 & 322.5 & 2.57 & 1.80 \\
\hline
\end{tabular}


Table 2 (continued)

\begin{tabular}{|c|c|c|c|c|c|c|c|c|}
\hline 1 & 2 & 3 & 4 & 5 & 6 & 7 & 8 & 9 \\
\hline 304.0 & 1.21 & 0.79 & 313.1 & 1.70 & 1.08 & 322.5 & 3.17 & 1.76 \\
\hline 308.1 & 1.59 & 0.95 & 313.5 & 2.37 & 1.18 & 327.0 & 2.79 & 2.02 \\
\hline 308.1 & 1.76 & 0.98 & 313.5 & 0.88 & 1.24 & 327.0 & 2.99 & 2.12 \\
\hline 308.1 & 1.91 & 0.98 & 313.5 & 1.76 & 1.22 & 327.0 & 3.20 & 2.04 \\
\hline \multicolumn{9}{|c|}{$\ln X_{2}=(6.61 \pm 0.54)-(3460 \pm 167) \cdot 1 / T ; \rho=0.9809 \%$} \\
\hline \multicolumn{9}{|c|}{ 2-methyl-5-(4-methylphenyl)-furan-3-carboxylic acid } \\
\hline \multicolumn{9}{|c|}{ Acetonitrile } \\
\hline 299.0 & 0.09 & 0.033 & 308.5 & 0.11 & 0.048 & 316.9 & 0.17 & 0.060 \\
\hline 299.0 & 0.09 & 0.033 & 312.1 & 0.16 & 0.053 & 316.9 & 0.19 & 0.063 \\
\hline 299.0 & 0.11 & 0.036 & 312.1 & 0.16 & 0.053 & 319.2 & 0.19 & 0.075 \\
\hline 299.2 & 0.07 & 0.035 & 313.5 & 0.17 & 0.058 & 319.2 & 0.14 & 0.074 \\
\hline 299.2 & 0.07 & 0.035 & 313.5 & 0.12 & 0.059 & 319.2 & 0.16 & 0.074 \\
\hline 299.2 & 0.12 & 0.036 & 313.5 & 0.16 & 0.057 & 320.5 & 0.21 & 0.076 \\
\hline 304.9 & 0.12 & 0.043 & 313.5 & 0.13 & 0.060 & 320.5 & 0.17 & 0.069 \\
\hline 304.9 & 0.10 & 0.041 & 313.5 & 1.25 & 0.058 & 320.5 & 0.18 & 0.073 \\
\hline 304.9 & 0.11 & 0.041 & 313.5 & 1.65 & 0.055 & 323.5 & 0.23 & 0.079 \\
\hline 308.5 & 0.10 & 0.047 & 314.1 & 1.65 & 0.061 & 323.5 & 0.18 & 0.080 \\
\hline 308.5 & 0.11 & 0.046 & 316.9 & 1.25 & 0.060 & 323.5 & 0.23 & 0.078 \\
\hline \multicolumn{9}{|c|}{$\ln X_{2}=(3.32 \pm 0.56)-(3382 \pm 175) \cdot 1 / T ; \rho=0.9800 \%$} \\
\hline \multicolumn{9}{|c|}{ Acetone } \\
\hline 293.2 & 0.40 & 0.29 & 299.8 & 0.38 & 0.33 & 313.4 & 0.90 & 0.49 \\
\hline 293.2 & 0.46 & 0.29 & 299.8 & 0.57 & 0.31 & 313.4 & 1.04 & 0.48 \\
\hline 293.2 & 0.55 & 0.28 & 299.8 & 0.75 & 0.31 & 313.4 & 1.08 & 0.49 \\
\hline 293.5 & 0.32 & 0.28 & 303.5 & 0.53 & 0.38 & 315.5 & 0.66 & 0.55 \\
\hline 293.5 & 0.40 & 0.27 & 303.5 & 0.75 & 0.38 & 315.5 & 0.93 & 0.55 \\
\hline 293.5 & 0.56 & 0.28 & 304.7 & 0.58 & 0.37 & 315.5 & 1.09 & 0.54 \\
\hline 296.2 & 0.40 & 0.29 & 304.7 & 0.68 & 0.37 & 319.7 & 0.78 & 0.60 \\
\hline 296.2 & 0.50 & 0.30 & 304.7 & 0.70 & 0.37 & 319.7 & 1.02 & 0.59 \\
\hline 296.2 & 0.62 & 0.30 & 308.6 & 0.58 & 0.44 & 322.6 & 1.06 & 0.62 \\
\hline 298.8 & 0.47 & 0.30 & 308.6 & 0.65 & 0.46 & 322.6 & 1.21 & 0.63 \\
\hline 298.8 & 0.70 & 0.30 & 308.6 & 0.83 & 0.46 & 322.6 & 1.24 & 0.63 \\
\hline \multicolumn{9}{|c|}{$\ln X_{2}=(3.54 \pm 0.47)-(2775 \pm 144) \cdot 1 / T ; \rho=0.9794 \%$} \\
\hline \multicolumn{9}{|c|}{ Isopropanol } \\
\hline 299.5 & 0.23 & 0.20 & 304.0 & 0.42 & 0.23 & 313.1 & 0.42 & 0.33 \\
\hline 299.5 & 0.36 & 0.19 & 306.7 & 0.36 & 0.26 & 313.1 & 0.59 & 0.34 \\
\hline 299.5 & 0.49 & 0.19 & 306.7 & 0.39 & 0.27 & 313.5 & 0.76 & 0.35 \\
\hline 302.5 & 0.35 & 0.23 & 306.7 & 0.45 & 0.26 & 313.5 & 0.57 & 0.34 \\
\hline 302.5 & 0.42 & 0.23 & 308.6 & 0.48 & 0.27 & 313.5 & 0.48 & 0.34 \\
\hline 303.7 & 0.25 & 0.23 & 308.6 & 0.51 & 0.28 & 317.9 & 0.43 & 0.40 \\
\hline 303.7 & 0.36 & 0.22 & 308.6 & 0.52 & 0.29 & 317.9 & 0.47 & 0.40 \\
\hline 303.7 & 0.39 & 0.23 & 308.6 & 0.65 & 0.29 & 322.5 & 0.83 & 0.45 \\
\hline 304.0 & 0.30 & 0.23 & 308.6 & 0.67 & 0.27 & 322.5 & 0.92 & 0.45 \\
\hline 304.0 & 0.33 & 0.23 & 313.1 & 0.41 & 0.35 & 322.5 & 1.21 & 0.46 \\
\hline \multicolumn{9}{|c|}{$\ln X_{2}=(5.92 \pm 0.43)-(3639 \pm 132) \cdot 1 / T ; \rho=0.9908 \%$} \\
\hline \multicolumn{9}{|c|}{ Ethylacetate } \\
\hline 293.6 & 0.25 & 0.26 & 312.4 & 0.48 & 0.40 & 318.4 & 0.95 & 0.51 \\
\hline 293.6 & 0.30 & 0.25 & 312.4 & 0.50 & 0.41 & 321.0 & 0.59 & 0.49 \\
\hline 297.1 & 0.32 & 0.28 & 313.0 & 0.46 & 0.43 & 321.0 & 0.61 & 0.48 \\
\hline 297.1 & 0.40 & 0.27 & 313.0 & 0.46 & 0.45 & 321.0 & 0.64 & 0.48 \\
\hline 303.8 & 0.37 & 0.32 & 313.0 & 0.67 & 0.43 & 323.0 & 0.79 & 0.53 \\
\hline 303.8 & 0.38 & 0.35 & 313.4 & 0.48 & 0.45 & 323.0 & 0.93 & 0.57 \\
\hline 303.8 & 0.52 & 0.33 & 313.4 & 0.59 & 0.45 & 323.0 & 0.07 & 0.54 \\
\hline 307.9 & 0.38 & 0.37 & 316.0 & 0.58 & 0.45 & 327.6 & 0.68 & 0.60 \\
\hline 307.9 & 0.40 & 0.36 & 316.0 & 0.59 & 0.44 & 327.6 & 0.71 & 0.59 \\
\hline 309.0 & 0.46 & 0.36 & 316.0 & 0.75 & 0.47 & 327.6 & 0.74 & 0.57 \\
\hline 309.0 & 0.46 & 0.36 & 318.4 & 0.50 & 0.49 & 333.0 & 0.80 & 0.70 \\
\hline
\end{tabular}


Table 2 (continued)

\begin{tabular}{|c|c|c|c|c|c|c|c|c|c|}
\hline 1 & 2 & 3 & 4 & 5 & 6 & 7 & 8 & 9 \\
\hline 312.4 & 0.44 & 0.39 & 318.4 & 0.82 & 0.52 & 333.0 & 1.30 & 0.69 \\
\hline \multicolumn{7}{|c|}{$\ln X_{2}=(2.44 \pm 0.43)-(2475 \pm 133) \cdot 1 / T ; \rho=0.9757 \%$} \\
\hline \multicolumn{7}{|c|}{ Benzene } \\
\hline 299.3 & 0.05 & 0.032 & 313.1 & 0.08 & 0.055 & 318.4 & 0.12 & 0.084 \\
\hline 299.3 & 0.04 & 0.031 & 313.1 & 0.11 & 0.065 & 322.5 & 0.12 & 0.086 \\
\hline 299.3 & 0.06 & 0.028 & 313.5 & 0.11 & 0.057 & 322.5 & 0.13 & 0.11 \\
\hline 303.5 & 0.06 & 0.036 & 313.5 & 0.19 & 0.068 & 322.5 & 0.14 & 0.11 \\
\hline 303.5 & 0.04 & 0.036 & 313.5 & 0.16 & 0.062 & 323.0 & 0.13 & 0.087 \\
\hline 303.5 & 0.10 & 0.037 & 317.9 & 0.14 & 0.084 & 323.0 & 0.19 & 0.098 \\
\hline 308.5 & 0.09 & 0.055 & 317.9 & 0.15 & 0.072 & 323.0 & 0.26 & 0.097 \\
\hline 308.5 & 0.09 & 0.047 & 317.9 & 0.25 & 0.078 & 327.0 & 0.16 & 0.11 \\
\hline 308.5 & 0.14 & 0.052 & 318.4 & 0.09 & 0.070 & 327.0 & 0.24 & 0.13 \\
\hline 313.1 & 0.07 & 0.070 & 318.4 & 0.10 & 0.076 & 327.0 & 0.27 & 0.13 \\
\hline \multicolumn{7}{|l|}{$\ln X_{2}=(8.2 \pm 1.2)-(4882 \pm 368) \cdot 1 / T ; \rho=0.9638 \%$} & & \\
\hline
\end{tabular}

Table 3

Standard enthalpies of fusion for 2-methyl-5-phenylfuran-3-carboxylic acid and 2-methyl-5-(4-methylphenyl)-furan-3-carboxylic acid at their fusion temperatures

\begin{tabular}{|c|c|c|c|c|}
\hline \multicolumn{7}{|c|}{$\Delta m_{\text {vap }}, \mathrm{g}$} & $S, \mathrm{~K} \cdot \mathrm{s}$ & $Q_{\text {vap }}, \mathrm{J}$ & $\Delta_{\text {fus }} H, \mathrm{~kJ} / \mathrm{mol}$ \\
\hline \multicolumn{7}{|c|}{ 2-Methyl-5-phenyl furan-3-carboxylic acid } \\
\hline \multicolumn{7}{|c|}{$M=202.21 \mathrm{~g} / \mathrm{mol} ; T_{\text {fus }}=458.1 \pm 0.5 \mathrm{~K} ; K=0.04215 \mathrm{~J} / \mathrm{K} \cdot \mathrm{s}$} \\
\hline 0.2003 & 0.0049 & 795.3 & 2.018 & 31.8 \\
\hline 0.2008 & 0.0047 & 805.2 & 1.954 & 32.2 \\
\hline \multicolumn{7}{|c|}{0.0035} & 798.6 & 1.432 & 33.2 \\
\hline 0.1786 & $2-$ Methyl-5-(4-methylphenyl)-furan-3-carboxylic acid \\
\hline 0.1981 & $M=216.24 \mathrm{~g} / \mathrm{mol} ; T_{\text {fus }}=507.2 \pm 1.2 \mathrm{~K} ; K=0.04951 \mathrm{~J} / \mathrm{K} \cdot \mathrm{s}$ \\
\hline 0.1998 & 0.0164 & 673.2 & 6.657 & 32.40 \\
\hline
\end{tabular}

Enthalpies of fusion for the acids were determined by differential thermal analysis. The noticeable loss of weight samples is observed in derivatograms. The losses are caused by partial evaporation of the samples during fusion. Therefore, when calculating heat transfer coefficient $K \quad(\mathrm{~J} / \mathrm{K} \cdot \mathrm{s})$, which determines the correspondence between the heat efficiency of the process and its peak area under DTA curve, and when calibrating the derivatograph cell, we take into account the correction for the sample weight loss caused by partial evaporation $\Delta m_{v a p}$ for the period of peak area $S(\mathrm{~K} \cdot \mathrm{s})$ determination under the DTA curve (Eq. (2)).

$$
K \cdot S=Q_{\text {fus }}+Q_{\text {vap }}=m_{o} \cdot \Delta H_{f u s}+\Delta m_{\text {vap }} \cdot \Delta H_{\text {vap }}
$$
where $Q_{\text {fus }}$ and $Q_{\text {vap }}$ - quantity of heat absorbed during fusion and evaporation, respectively, $\mathrm{J} ; m_{0}$-sample weight corresponding to the beginning temperature of fusion $T_{f u s}, \mathrm{~g} ; \Delta_{f u s} H$ and $\Delta_{v a p} H$ - specific enthalpy of fusion and evaporation, respectively, at the fusion temperature, $\mathrm{kJ} / \mathrm{g}$.

Enthalpies of evaporation were determined according to the rate of the samples evaporation from the derivatograph cell within the temperature range from their full fusion to the beginning of thermooxidative destruction. The results of enthalpies and entropies of fusion are represented in Table 3.

Standard enthalpy and entropy of fusion for 2-methyl-phenylfuran-3-carboxylic and 2-methyl-5(4-methylphenyl)-furan-3-carboxylic acids were determined according to DTA data at their fusion temperatures of 458.1 and $507.2 \mathrm{~K}$, respectively. To recalculate thermodynamic properties for $298 \mathrm{~K}$ according to Kirchhoff equation we have to know the difference between heat capacity of the compounds in the solid and liquid state within the temperature range from the fusion temperature to $298 \mathrm{~K}$. In our case the temperature range is narrow (up to $210 \mathrm{~K}$ ) and it is enough to know the difference in heat capacities corresponding to the middle of the ranges. Whereas the heat capacities of the synthesized acids were not determined experimentally, it was necessary to calculate the difference between heat capacities. J. Chickos et al. [14] represents semiempirical Eq. (3) to calculate the enthalpy of fusion at $298 \mathrm{~K}$ taking into account molar heat capacity of the compounds in the solid $C_{p}{ }^{0}\left({ }_{(c)}\right.$ and liquid $C_{p}{ }^{0}(l)$ states:

$$
\begin{aligned}
\Delta_{f u s} H^{0}{ }_{298} & =\Delta_{f u s} H_{\text {Tfus }}^{0}+\left(0.15 C_{p(c)}^{0}-0.26 C_{p(l)}^{0}-\right. \\
& -9.83)\left(T_{f u s}-298\right)
\end{aligned}
$$


According to [19] the molar heat capacity was calculated by additive scheme using group contributions given in the same paper. To calculate the entropy of fusion at $298 \mathrm{~K} \mathrm{Eq}$. (3) was rewritten in a similar way:

$$
\begin{aligned}
& \Delta_{f u s} S_{298}^{0}=\Delta_{f u s} S_{\text {Tfus }}^{0}+\left(0.15 C_{p}^{0}(c)-\right. \\
& \left.-0.26 C_{p}^{0}(l)-9.83\right) \ln \left(T_{f u s} 298\right)
\end{aligned}
$$

On the other hand, the ratio of standard entropy to the heat capacity of fusion at their fusion point was found to be constant $( \pm 7 \%)$ [12] for more than 100 organic compounds (aliphatic, alicyclic, aromatic hydrocarbons and their substitutes - alcohols, aldehydes, ketones, acids, halogen- and nitroderivatives $[20,21])$.

$$
\left(\frac{\Delta_{f u s} S^{0}}{\Delta_{\text {fus }} C p^{0}}\right)_{T_{\text {fus }}}=\left(\frac{\Delta_{\text {fus }} H^{0}}{T_{\text {fus }} \cdot \Delta_{\text {fus }} C p^{0}}\right)_{T_{\text {fus }}}=1.35 \pm 0.11
$$

In combination with classical thermodynamic equations the expression for calculation of the molar enthalpies and entropies of fusion became as follows:

$$
\begin{aligned}
\Delta_{\text {fus }} H^{0}{ }_{298} & =\Delta_{\text {fus }} H_{T_{\text {fus }}}^{0}-\Delta_{\text {fus }} C p^{0} \cdot\left(T_{\text {fus }}-298\right)= \\
& =\Delta_{\text {fus }} H_{T_{\text {fus }}}^{0} \cdot \frac{0.35 \cdot T_{\text {fus }}+298}{1.35 \cdot T_{\text {fus }}}
\end{aligned}
$$

$$
\begin{gathered}
\Delta_{\text {fus }} S^{0}{ }_{298}=\Delta_{\text {fus }} S_{T_{\text {fus }}}^{0}-\Delta_{\text {fus }} C p^{0} \cdot \ln \frac{T_{\text {fus }}}{298}= \\
=\Delta_{\text {fus }} S_{T_{\text {fus }}}^{0} \cdot \frac{1.35-\ln \frac{T_{f u s}}{298}}{1.35}
\end{gathered}
$$

Enthalpies and entropies of fusion of the investigated acids at $298 \mathrm{~K}$ which were recalculated from the temperatures of fusion by both methods are represented in Table 4. One can see that calculated values are close and indistinguishable within the accuracy of their determination. However, according to Eqs. (3) and (4), the heat capacities were calculated in regard to group contributions at $298 \mathrm{~K}$, and according to Eqs. (6) and (7) at the temperature of fusion. As mentioned above, it is necessary to use the heat capacity at average temperatures within the range from fusion temperature to $298 \mathrm{~K}$. Therefore, we think that average values of thermodynamic

\begin{tabular}{|c|c|c|c|}
\hline$\Delta_{f u s} H^{0}$ & $\Delta_{f u s} S^{0}=\Delta_{f u s} H^{0} / T_{\text {fus }}$ & $\Delta_{\text {fus }} H^{0}$ & $\Delta_{f u s} S^{0}$ \\
\hline \multicolumn{2}{|c|}{ at $T_{\text {fus }}$ (experimental) } & \multicolumn{2}{|c|}{ at $298 \mathrm{~K}$ (calculated) } \\
\hline \multicolumn{4}{|c|}{ 2-Methyl-5-phenylfuran-3-carboxylic acid } \\
\hline \multirow{2}{*}{$32.4 \pm 1.8$} & \multirow{2}{*}{$70.7 \pm 1.9$} & $22.5 \pm 2.3 *($ Eq. 3) & $44.0 \pm 2.4 *(\mathrm{Eq} .4)$ \\
\hline & & $24.0 \pm 1.9$ (Eq. 6$)$ & $48.2 \pm 2.0(\mathrm{Eq} .7)$ \\
\hline & Mean value: & $23.3 \pm 3.0$ & $46.1 \pm 3.1$ \\
\hline \multicolumn{4}{|c|}{ 2-Methyl-5-(4-methylphenyl)-furan-3-carboxylic acid } \\
\hline \multirow{2}{*}{$32.69 \pm 0.98$} & \multirow{2}{*}{$65.5 \pm 1.5$} & $19.0 \pm 1.6 *($ Eq. 3) & $37.7 \pm 2.1 *(\mathrm{Eq} .4)$ \\
\hline & & $22.7 \pm 1.1($ Eq. 6) & $39.1 \pm 1.6(\mathrm{Eq} .7)$ \\
\hline & Mean value: & $20.8 \pm 1.9$ & $38.4 \pm 2.6$ \\
\hline
\end{tabular}
properties calculated by two methods are the most accurate ones. Using enthalpies and entropies we calculated Gibbs energy of solubility for the investigated acids in organic solvents (Table 5).

Table 4

Standard enthalpies $(\mathrm{kJ} / \mathrm{mol})$ and entropies $(\mathrm{J} / \mathrm{mol} \cdot \mathrm{K})$ of fusion for the investigated acids at $298 \mathrm{~K}$

Note: *errors of heat capacity values for the solid and liquid state were calculated as a sum of group contributions, the value of which was $30 \mathrm{~J} / \mathrm{mol} \cdot \mathrm{K}[19]$.

Table 5

Thermodynamic solubility of 2-methyl-5-phenylfuran-3-carboxylic acid and 2-methyl-5-(4-methylphenyl)-furan-3-carboxylic acid in organic solvent at $298 \mathrm{~K}$

\begin{tabular}{|l|c|c|c|c|c|c|}
\hline \multirow{2}{*}{ Solvent } & $\Delta_{\text {sol }} \mathrm{H}$ & $\Delta_{\text {mix }} \mathrm{H}$ & $\Delta_{\text {so }} S$ & $\Delta_{\text {mix }} S$ & $\Delta_{\text {sol }} G$ & \multicolumn{2}{c|}{$\mathrm{J} / \mathrm{mol} \cdot \mathrm{K}$} \\
\cline { 2 - 7 } & \multicolumn{7}{|c|}{$\mathrm{kJ} / \mathrm{mol} \cdot \mathrm{K}$} & $\Delta_{\text {mix }} G$ \\
\hline \multicolumn{7}{|c|}{ 2-Methyl-5-phenylfuran-3-carboxylic acid } \\
\hline Acetonitrile & $26.7 \pm 1.1$ & $3.4 \pm 3.2$ & $41.0 \pm 3.5$ & $-5.1 \pm 4.7$ & $14.5 \pm 3.7$ & $4.9 \pm 5.7$ \\
\hline Acetone & $16.61 \pm 0.36$ & $-6.7 \pm 3.0$ & $25.8 \pm 1.2$ & $-20.3 \pm 3.3$ & $8.9 \pm 1.3$ & $-0.7 \pm 4.4$ \\
\hline Isopropanol & $22.68 \pm 0.85$ & $-0.62 \pm 3.0$ & $41.9 \pm 2.7$ & $-4.2 \pm 4.1$ & $10.2 \pm 2.8$ & $0.63 \pm 5.1$ \\
\hline Ethylacetate & $17.24 \pm 0.34$ & $-6.1 \pm 3.0$ & $26.8 \pm 1.1$ & $-19.3 \pm 3.3$ & $9.2 \pm 1.2$ & $-0.3 \pm 4.4$ \\
\hline Benzene & $28.8 \pm 1.4$ & $5.5 \pm 3.3$ & $55.0 \pm 4.5$ & $8.9 \pm 5.5$ & $12.4 \pm 4.7$ & $4.5 \pm 6.4$ \\
\hline \multicolumn{7}{|c|}{2 -Methyl-5-(4-methylphenyl)-furan-3-carboxylic acid } \\
\hline Acetonitrile & $28.1 \pm 1.5$ & $7.3 \pm 2.4$ & $27.6 \pm 4.7$ & $-10.8 \pm 5.4$ & $19.9 \pm 4.9$ & $10.5 \pm 5.9$ \\
\hline Acetone & $23.1 \pm 1.2$ & $2.3 \pm 2.1$ & $29.4 \pm 3.9$ & $-9.0 \pm 4.7$ & $14.3 \pm 4.1$ & $4.9 \pm 5.1$ \\
\hline Isopropanol & $30.2 \pm 1.1$ & $9.4 \pm 2.2$ & $49.2 \pm 3.6$ & $10.8 \pm 4.4$ & $15.5 \pm 3.8$ & $6.2 \pm 4.9$ \\
\hline Ethylacetate & $20.6 \pm 1.1$ & $-0.2 \pm 2.2$ & $20.3 \pm 3.6$ & $-18.1 \pm 4.4$ & $14.6 \pm 3.8$ & $5.2 \pm 4.9$ \\
\hline Benzene & $40.6 \pm 3.1$ & $19.8 \pm 3.6$ & $68.2 \pm 9.9$ & $30 \pm 10$ & $20 \pm 10$ & $11 \pm 11$ \\
\hline
\end{tabular}




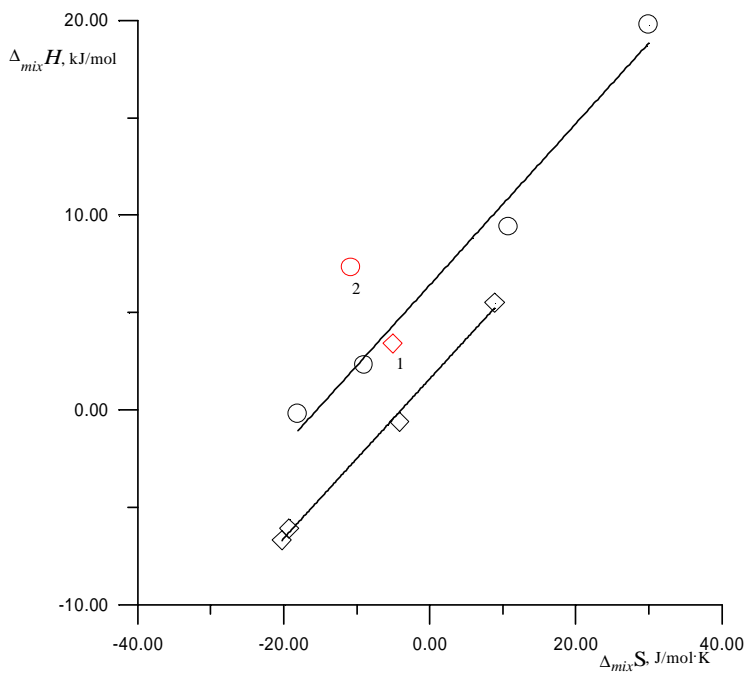

Fig. 2. Dependence between partial molar enthalpy and entropy of solubility for the investigated compounds in different solvents: 2-methyl-5-phenylfuran-3-carboxylic acid (circle) and 2-methyl5-(4-methylphenyl)-furan-3-carboxylic acid (diamond). Points 1 and 2 are for the investigated compounds in acetonitrile

We observe the straight-line relationship between the partial molar enthalpy and entropy of mixing of both acids with all solvents (except acetonitrile): for 2-methyl-5phenylfuran-3-carboxy acid (Fig. 2 and Eq. 8) and 2- methyl5-(4-methylphenyl)-furan-3-carboxy acid (Fig. 2 and Eq. 9).

$$
\begin{gathered}
\Delta_{m i x} H_{298}=0.414 \cdot \Delta_{m i x} S_{298}+6.40 ; R=0.983 \\
\Delta_{m i x} H_{298}=0.409 \cdot \Delta_{m i x} S_{298}+1.59 ; R=0.996
\end{gathered}
$$

This fact indicates the presence of mixing compensating effect, which depends on rearrangement of intermolecular interactions of the dissolved compound and the solvent. The investigated acids and solvents are capable of forming intermolecular hydrogen bonds with oxygen through the carboxy and hydroxy groups. In acetonitrile molecules the hydrogen bond is formed between hydrogen atom of hydroxy and nitrogen atom of nitrile groups. Obviously, this difference can be explained by the fact that the points of entropy dependence on the enthalpy of mixing are not situated on the straight lines, corresponding to the mixing compensating effect of these acids with other solvents.

\section{Conclusions}

Since the synthesis, purification, extraction, and application of investigated compounds take place mainly in the solutions, the obtained parameters can be directly used to calculate the optimal energy consumption of the corresponding industrial processes.

\section{References}

[1] Fürstner A., Castanet A., Radkowski K., Lehmann C.: J. Org. Chem., 2003, 68, 1521. https://doi.org/10.1021/jo026686q
[2] Holla B., Akberali P., Shivananda M.: Farmaco, 2000, 55, 256. https://doi.org/10.1016/S0014-827X(00)00030-6

[3] Williams D., Lee M.-R., Song Y.-A. et al.: J. Am. Chem. Soc., 2007, 129, 9258. https://doi.org/10.1021/ja072817z

[4] Obushak N., Gorak Yu., Matiichuk V., Lytvyn R.: Russ. J. Org. Chem., 2008, 44, 1689.

https://doi.org/10.1134/S1070428008110213

[5] Obushak N., Gorak Yu., Matiichuk V., Lytvyn R.: Russ. J. Org. Chem., 2009, 45, 541. https://doi.org/10.1134/S1070428009090103

[6] Obushak N., Lesyuk A., Gorak Yu., Matiichuk V.: Russ. J. Org. Chem., 2009, 45, 1375.

https://doi.org/10.1134/S1070428009090103

[7] Obushak N., Lesyuk A., Ganushchak N. et al.: J. Org. Chem. USSR (Engl. Transl.), 1986, 22, 2093.

[8] Obushak N., Ganushchak N., Lesyuk A. et al.: J. Org. Chem. USSR (Engl. Transl.), 1990, 26, 748.

[9] Zhu J., Bienayme H. (Eds.): Multicomponent Reactions. WileyVCH, Weinheim 2005.

[10] Sobechko I., Van-Chin-Syan Yu., Gorak Yu. et al:: Rus. J.

Phys. Chem., 2015, 89, 919.

https://doi.org/10.1134/S003602441506028X

[11] Sobechko I., Gorak Yu., Van-Chin-Syan Yu. et al.: Izv. Vys. Ucheb. Zaved., 2015, 58, 45.

[12] Sobechko I., Prokop R., Gorak Yu. et al.: Voprosy Khimii i Khim. Techn., 2013, 4, 12.

[13] Sobechko I.: Voprosy Khimii i Khim. Techn., 2014, 5-6, 48.

[14] Serheyev V.: Chem. Chem. Technol., 2012, 6, 15.

[15] Serheyev V.: Chem. Chem. Technol., 2015, 9, 1.

[16] Serheyev V.: Rus. J. Phys. Chem., 2016, 90, 575.

https://doi.org/10.1134/S0036024416030274

[17] Han S., Meng L., Du C. et al.: J. Chem. Thermodyn., 2017, 97, 17. https://doi.org/10.1016/j.jct.2015.11.025

[18] Li X., Du C., Cong Y., Zhao H.: J. Chem. Thermodyn., 2017, 104, 189. https://doi.org/10.1016/j.jct.2016.09.033

[19] Chickos J., Acree W. Jr.: J. Phys. Chem. Ref. Data, 2003, 32, 519. https://doi.org/10.1063/1.1529214

[20] http://webbook.nist.gov (March 18th, 2015)

[21] Vasil'ev I., Petrov V.: Thermodynamicheskie Svoistva Kislorod-soderzhashih Organicheskih Veshestv. Khimiya, Leningrad 1984.

Received: N ovember 25, 2016 / Revised: December 15, 2016 / Accepted: J anuary 27, 2017

\section{ТЕРМОДИНАМІЧНІ ВЛАСТИВОСТІ РОЗЧИННОСТІ \\ 2-МЕТИЛ-5-АРИЛФУРАН-3-КАРБОНОВИХ КИСЛОТ В ОРГАНІЧНИХ РОЗЧИННИКАХ}

Анотація. Досліджені температурні залежності розчинності обох кислот в ацетонітрилі, диметилкетоні, ізо-пропанолі, етилацетаті та бензені. Результати представлені лінійною формою рівняння Шредера, за яким визначені ентальпії, ентропії та енергії Гіббса розчинності за 298 К. Теплоти плавлення кислот визначені методом диференційно-термічного аналізу, за якими розраховані ентальпії, ентропії та енергії Гіббса змішування. Виявлений компенсаиійний ефект змімування для всіх розчинників, що містять карбонільну групу.

Ключові слова: ентальпія, ентропія та енергія Гіббса розчинності, змішування і плавлення, 2-метил-5-фенілфуран-3карбонова кислота та 2-метил-5-(4-метилфеніл)-фуран-3карбонова кислота. 